\title{
The value of deep learning-based computer aided diagnostic system in improving diagnostic performance of rib fractures in acute blunt trauma
}

Hui Tan

Affiliated Hospital of Shaanxi University of Chinese Medicine

Hui Xu

McMaster University Department of Psychiatry and Behavioural Neurosciences

Nan Yu

Shaanxi University of Chinese Medicine

Yong Yu

Shaanxi University of Chinese Medicine

Haifeng Duan

Shaanxi University of Chinese Medicine

Qiuju Fan ( $\nabla$ fanqiuju858700@gmail.com )

Shaanxi University of Chinese Medicine

Zhanyu Tian

Shaanxi University of Chinese Medicine

\section{Research article}

Keywords: Rib Fracture, Deep Learning, Computer Aided Diagnostic System, Diagnostic Performance,

Reading Time

Posted Date: April 13th, 2021

DOI: https://doi.org/10.21203/rs.3.rs-413049/v1

License: (c) (1) This work is licensed under a Creative Commons Attribution 4.0 International License. Read Full License 


\section{Abstract \\ Purpose}

To evaluate the value of a deep learning-based computer-aided diagnostic system (DL-CAD) in improving the diagnostic performance of acute rib fractures in patients with chest trauma.

\section{Methods}

CT images of 214 patients with acute blunt chest trauma were retrospectively analyzed by two interns and two attending radiologists independently firstly and then with the assistance of a DL-CAD one month later, in a blinded and randomized manner. The consensus diagnosis of fib fracture by another two senior thoracic radiologists was regarded as reference standard. The rib fracture diagnostic sensitivity, specificity, positive predictive value, diagnostic confidence and mean reading time with and without DL-CAD were calculated and compared.

\section{Results}

There were 680 rib fracture lesions confirmed as reference standard among all patients. The diagnostic sensitivity and positive predictive value of interns were significantly improved from $(68.82 \%, 84.50 \%)$ to $(91.76 \%, 93.17 \%)$ with the assistance of DL-CAD, respectively. Diagnostic sensitivity and positive predictive value of interns assisted by DL-CAD were comparative to those of attendings aided by DL-CAD $(94.56 \%$, $86.47 \%)$ or not aided $(95.67 \%, 93.83 \%)$, respectively. In addition, when radiologists were assisted by DL-CAD, the mean reading time was significantly reduced and diagnostic confidence was significantly enhanced.

\section{Conclusions}

DL-CAD improves the diagnostic performance of acute rib fracture in chest trauma patients, which increases the diagnostic confidence, sensitivity and positive predictive value for radiologists. DC-CAD can advance the diagnostic consistency of radiologists with different experiences.

\section{Background}

Acute rib fracture (ARF) is a common traumatic fracture of the chest in clinical work. Timely and accurate diagnosis of ARF is not only of great value for clinical treatment but also an important indicator for forensic disability classification which can reduce unnecessary medical disputes. ${ }^{1-4} \mathrm{X}$-ray has poor diagnostic efficiency for rib fracture, due to its low contrast resolution and overlapping structures. Multi-detector spiral CT (MDCT) is the most sensitive imaging modality for the diagnosis of ARF at present. ${ }^{5,6}$ Nowadays, the annual growth rate of medical imaging data in China is about $30 \%$, while the annual growth rate of radiologists is only $4.1 \%$ which imposes huge burden for radiologists to process more image data. ${ }^{7,8}$ Under 
heavy workload, manual interpretation which relies on physician's experience is error-prone and often leads to a high rate of misdiagnosis and missed diagnosis. ${ }^{9,10}$

The application of deep learning-based computer aided diagnostic (DL-CAD) system in medical imaging is an effective method to resolve the current shortage of radiologists. ${ }^{11}$ However, the clinical application of DLCAD traditionally focused on the detection of pulmonary nodules and the distinction between benign and malignant tumors, ${ }^{12-14}$ the clinical application of DL-CAD in the detection of ARF has not been reported and its role in the diagnosis of ARF needs to be verified and validated through clinical experiments.

Therefore, our study aimed to explore the influence of DL-CAD on diagnostic confidence, diagnostic quality and reading time for ARF detection in different reading modes.

\section{Methods And Materials}

\section{Participants}

A total of 214 cases of ARFs diagnosed by chest CT examination due to acute blunt chest trauma were collected retrospectively from July 2018 to February 2019 in our hospital, including 123 males and 91

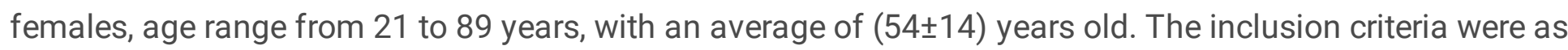
follows: 1) A clear history of acute chest trauma; 2) certain or suspected rib fractures were reported by the radiological diagnoses. Exclusion criteria: Image quality undiagnostic due to motion artifacts, ${ }^{15}$ unconsciousness or lack of self-control, etc. The Affiliated Hospital of Shaanxi University of Chinese Medicine institutional review board reviewed and approved the protocol and provided continuing oversight. All participants provided written informed consent.

\section{CT Examination}

All cases were scanned with a 64-row spiral CT (Discovery HD 750, GE Healthcare). Scan position: supine position with upper arm lifted (some patients were naturally placed on both sides of the body due to injury in the shoulder or upper limb). Scanning range: from the thoracic opening to the 12th rib lower edge. Scanning parameters: tube voltage: $120 \mathrm{kVp}$, tube current: $20 \mathrm{~mA}-500 \mathrm{~mA}$, pitch: 0.85 , reconstruction convolution kernel: Standard or Bone, slice thickness: $1.25 \mathrm{~mm}$.

\section{DL-CAD System}

A commercial and easy-to-use DL-CAD system (InferRead CT Bone Research, Infervision, Beijing, China), which extracted image features via an artificial convolutional neural network to automatically detect rib fractures were used in our study. An information list containing all detected rib fractures for each patient were provided by the DL-CAD, and the location of each fracture on the CT images was labeled with a box.

\section{Reading Experiment}

2 intern radiologists (who have less than 1 year of experience) and 2 attending radiologists ( who have 7 years of experience) participated in this reading experiment. The task was performed in two reading 
sessions at an interval of 4 weeks apart. At each session, all 214 cases were randomized into 7 groups, including 31 patients in groups $1,3,5$, and 7 , and 30 patients in groups 2,4 , and 6 . All data sets were presented to readers in randomized order, and orders were different for every reader. In order to reduce the adverse impact from fatigue, which was usually caused by long-time consecutive reading work, the readers performed one group of reading experiment per day and thus they finished each session in a week. All readers interpreted all the cases on a picture archiving and communication system (PACS) independently in the first session. And after a memory washout period of 4 weeks, the second session were implemented, in which the reader re-interpreted all cases with the assistance of DL-CAD in concurrent reading mode. During the reading procedure, the readers could adjust the window width/level and zoom in/out, or use maximum intensity projection (MIP) or volume rendering (VR) if needed.

In both reading sessions, all readers were instructed to focus on detecting rib fracture. Considering that each rib has a possibility of fracture, 24 ribs for each patient were evaluated. And a 5-point Likert-scale was used

to evaluate the diagnostic confidence of each rib: 1 , definitely absent; 2 , probably absent; 3 , indeterminate; 4 , probably present; 5 , definitely present. For each patient, the fracture locations and diagnostic confidence score of each rib and the time-consumption for reading procedure were recorded. All readers had received DL-CAD system knowledge training before reading.

\section{Gold Standard}

Two senior radiologists (Mr. JIA and Mr. DUAN, both with more than 15 years of experience in chest CT diagnosis) reviewed all the cases and their consensus diagnoses were referred as surrogate gold standard. Additionally, in order to evaluate the diagnostic performance of complete rib fracture (CRF) and occult rib fracture (ORF), each fracture was classified as either complete or occult by the two senior radiologists. A CRF was confirmed if the fracture line run through the entire cortical bone with the cortex continuity completely interrupted. An ORF was referred to the bone density increasing, folding, warping, partial unconnected at external and/or internal bone cortex. ${ }^{16}$ In order to improve diagnostic accuracy, the electronic medical record and follow up CT examination images would be reviewed in this gold standard session if needed.

\section{Statistical Analysis}

The diagnostic sensitivity, specificity, positive predictive value (PPV), diagnostic confidence and average time-consumption per case were calculated and compared between two reading sessions. Receiver operating characteristics (ROC) analysis was performed and area under the receiver operating characteristic curve (AUC) was calculated and compared. The paired sample t-test was used to compare quantitative data, Chi-square test was used to compare the Constituent ratio data, and nonparametric Wilcoxon test was used to compare the ranked data. The $P$ value of less than 0.05 was considered statistically significant. Statistical analysis was performed using SPSS ${ }^{\circledR}$ Statistics 19.0 (IBM Corporation, Armonk, NY; formerly SPSS Inc., Chicago, IL).

\section{Results}




\section{Patients' Characteristics}

A total of 214 patients with 680 ARFs were confirmed by the two senior radiologists as the surrogate gold standard, including 529 CRFs and 151 ORFs (Table 1).

\section{Comparison of Diagnostic Performance}

468 rib fractures, including 396 CRFs and 72 ORFs, were accurately detected by the interns independently. The sensitivity, specificity and PPV were $68.82 \%, 96.25 \%$ and $84.50 \%$, respectively. When assisted by DLCAD, 624 rib fractures, including 503 CRFs and 121 ORFs, were accurately detected. The sensitivity and PPV significantly increased to $91.76 \%$ and $93.17 \%(P<0.05)$, respectively, while the specificity remained at similar with a value of $97.46 \%$. Among them, the sensitivity of ORFs increased from $47.68 \%$ to $80.13 \%(P<0.05)$. ROC analysis exhibited that the AUC was improved from 0.925 to 0.977 (figure 1). By contrast, 588 rib fractures, including 476 CRFs and 112 ORFs, were accurately detected by the attendings independently. The sensitivity, specificity and positive predictive value (PPV) were $86.47 \% \varangle 97.55 \%$ and $93.83 \%$, respectively. When assisted by DL-CAD, 643 rib fractures, including 513 CRFs and 130 ORFs, were accurately detected. The sensitivity significantly increased to $94.56 \%(P<0.05)$, while the specificity and PPV remained similar, with a value of $98.47 \%$ and $95.67 \%$ respectively. Besides, the sensitivity of ORFs increased from $74.17 \%$ to 86.09\% ( $\mathrm{P}<0.05)$. AUC was also improved from 0.955 to 0.987 (Fig. 1). Additionally, with a substantial improvement of the sensitivity and PPV for the interns, no statistically significant difference was found between the interns and attendings in all observation parameters including sensitivity, specificity and PPV when assisted by DL-CAD, which indicated that the inter-observer consistency between interns and attendings was improved (Table 2).

\section{Comparison of Diagnostic Confidence}

The average diagnostic confidence scores of the interns in the first and second session were $3.69 \pm 1.12$ and $4.32 \pm 0.87$, respectively, with a statistically significant difference $(P<0.05)$. in particularly, the average diagnostic confidence scores of CRFs and ORFs were significantly improved from $3.94 \pm 1.09$ and $2.27 \pm 1.31$ to $4.45 \pm 0.79$ and $3.78 \pm 1.22$ respectively $(P<0.05)$. The ratio of 5-points confidence (definitely present) increased from 26.47\% (180 / 680) to 53.38\% (363 / 680) when interns were assisted by DL-CAD. In terms of attendings, a similar tendency was found. The average diagnostic confidence scores of the first and second session was $4.11 \pm 1.03$ and $4.54 \pm 0.72$, respectively, with a statistically significant difference $(\mathrm{P}<0.05)$. The average diagnostic confidence scores of CRFs and ORFs significantly increased from $4.26 \pm 1.00$ and $3.47 \pm 1.15$ to $4.65 \pm 0.63$ and $4.09 \pm 1.07$ respectively $(P<0.05)$. The ratio of 5-points confidence (definitely present) also significantly increased from 44.26\% (301 / 680) to 63.53\% (432 / 680) (Fig. 2, Fig. 3 and Table 3). Overall, compared with independent reading, both interns and attendings were significantly more confident in their diagnoses when using DL-CAD assistance.

\section{Comparison of Diagnostic Efficiency}

The average time-consumption per patient was $99.48 \pm 21.69 \mathrm{~s}$ when interns interpreted independently in the first session, which was reduced to $46.40 \pm 26.40 \mathrm{~s}$ with the assistance of DL-CAD in the second 
session, corresponding to a substantial decrease of $53.4 \%(P<0.05)$. Similarly, the average timeconsumption of the attendings in the first and second session was $65.96 \pm 17.08 \mathrm{~s}$ and $43.54 \pm 23.54 \mathrm{~s}$ respectively, which suggested a significant decrease of $34.0 \%$ was achieved when assisted by DL-CAD $(P<$ 0.05) (Fig. 4).

\section{Discussion}

Thoracic traumas comprise $10-15 \%$ of all traumas and are the causes of death in $25 \%$ of all fatalities due to trauma. Rib fractures are often implicated in blunt thoracic injury. A previous study suggests that patients with isolated rib fractures should be hospitalized if the number of fractured ribs is three or more. It also advocates that elderly patients with six or more fractured ribs should be treated in intensive care units due to high morbidity and mortality. ${ }^{17}$ Another clinical study indicates that the greater number of fractured ribs correlates with higher the mortality and morbidity rates. It is vital for the emergency clinical workflow to timely and accurately identify the presence of rib injuries that require urgent attention. ${ }^{18}$

CT examination is more effective than DR in detecting acute rib fracture. ${ }^{19,20}$ However, due to the subjective factors such as heavy workload, fatigue and inconsistent diagnostic experience, diagnosis of acute rib fracture with $\mathrm{CT}$ has a high misdiagnosis rate. ${ }^{20}$ At present, unlike fields such as lung cancer screening, where several studies about clinical application of DL-CAD have been reported, ${ }^{21-25}$ the research on the application of deep learning technology in fracture diagnosis mostly focuses on algorithms development. ${ }^{26}$ The impact of DL-CAD on the quality, confidence and efficiency for rib fractures diagnoses in clinical practice is still unclear.

In order to improve diagnostic quality, all radiological reports were generated by a 2-step review system in China which was firstly written by a junior radiologist (Intern or resident) with less experience, and then confirmed by a senior radiologist (attending or more senior) with more experience. In this study, to explore the impact of DL-CAD for novel and experienced radiologists, two interns and two attending radiologists interpreted all cases independently in a blind and random way without or with the help of DL-CAD in two sessions, and the impact of DL-CAD was analyzed.

The lack of diagnostic confidence usually leads to a more conservative assessment, and sufficient confidence helps to give clear suggestions for treatment, which could potentially reduce the excessive examination caused by follow-ups. ${ }^{27,28}$ It is worth noting that there are interpretation inconsistency between different diagnostic radiologists, especially for the ORFs, because of its inconspicuous image manifestations. ${ }^{29,30}$ In the process of clinical imaging diagnosis, it is necessary to closely combine the patient's history of trauma, including the exact location and time of trauma, or the occurrence of callus during reexamination. The results of our study exhibited that compared with independent diagnosis, the average diagnosis confidence of interns and attending doctors increased from $3.69 \pm 1.12$ and $4.11 \pm 1.03$ to $4.32 \pm 0.87$ and $4.54 \pm 0.72(P<0.05)$ with the assistance of DL-CAD in a concurrent reading mode. Besides, the diagnostic confidence of both CRF and ORF for interns and attendings were improved. Additionally, the ratio of highest diagnostic confidence (5-points) increased from $26.47 \%$ (180 / 680) to $53.38 \%(363 / 680)$ for interns, and from $44.26 \%$ (301/680) to $63.53 \%(432 / 680)$ for attendings respectively 
$(P<0.05)$. Those results indicated that the diagnostic confidence of radiologists did benefit from the assistance of DL-CAD system.

In terms of diagnostic quality, the sensitivity and PPV of interns in independent diagnosis were significantly lower than those of attending radiologists. The sensitivity, specificity and PPV was improved by the assistance of LD-CAD from $68.82 \%, 96.25 \%$ and $84.50-91.76 \%, 93.17 \%$ and $97.46 \%$ for interns, and from $86.47 \%, 97.55 \%$ and $93.83-94.56 \%(P<0.05), 98.47 \%$ and $95.67 \%$ for attendings. Besides, much more ORFs were detected with the assistance of DL-CAD for both interns and attendings. The ROC analysis also clearly exhibited a larger AUC for both interns and attendings when using DL-CAD. Those results suggested that the diagnostic quality of radiologists with different diagnostic experience could benefit from the use of DL-CAD. In addition, compared with attendings, a more substantial improvement was found for the interns. No statistically significant difference was found between the interns and attendings in all observation parameters including sensitivity, specificity and PPV when assisted by DL-CAD, which apparently resulted in a decrease of inter-observer difference. This result further evidenced that DL-CAD could significantly improve the diagnostic consistency of radiologists with different experiences. This may be because the deep learning algorithms had a higher detection accuracy and could reduce the doctors' dependence on diagnostic experience. As for the diagnostic efficiency, this study showed that the average timeconsumption per patient of interns and attendings both decreased significantly $(P<0.05)$ when they were assisted by DL-CAD. It was probably because interpreting radiologists could locate the fracture lesion more quickly and make a diagnostic conclusion with less hesitation, with the position information of fracture provided by DL-CAD system and a higher diagnostic confidence.

\section{Conclusions}

In conclusion, our study showed that DL-CAD system can enhance the diagnostic confidence and quality, improve the diagnostic consistency for doctors with different experiences, and reduce the time-consumption meanwhile.

\section{Declarations}

\section{Ethics approval and consent to participate}

This study was approved and consented by the Ethics Committee of Affiliated Hospital of Shaanxi University of Chinese Medicine. Informed consent was obtained from all individual participants included in the study.

\section{Consent for publication}

Not applicable.

\section{Availability of data and materials}


The datasets used and/or analyzed during the current study are available from the corresponding author on reasonable request.

\section{Competing interest}

The authors declare that they have no conflict of interest.

\section{Funding}

This study was supported by research grants from Subject Innovation Team of Shaanxi University of Chinese Medicine (2019-QN09).

\section{Authors' contributions}

H.T. and N.Y. conceived the study, designed the trial, and obtained research funding. Q.F., Z.T. supervised the conduct of the trial and data collection. Y.Y. and D.H. undertook recruitment of participating centers and patients and managed the data, including quality control. H.X. provided statistical advice on study design and analyzed the data; H.T. drafted the manuscript, and all authors contributed substantially to its revision. H.X. and Q.F. takes responsibility for the paper as a whole.

\section{Acknowledgements}

Not applicable

\section{References}

1. Glemser Philip A., Pfleiderer Michael, Heger Anna, et al. New bone post-processing tools in forensic imaging: a multi-reader feasibility study to evaluate detection time and diagnostic accuracy in rib fracture assessment. International Journal of Legal Medicine. 2017;131:1-8.

2. H. Ringl, M. Lazar, M. Töpker, et al. The ribs unfolded - a CT visualization algorithm for fast detection of rib fractures: effect on sensitivity and specificity in trauma patients. European Radiology. 2015;25:18651874.

3. Langdorf M. I., Medak A. J., Hendey G. W., et al. Prevalence and Clinical Import of Thoracic Injury Identified by Chest Computed Tomography but Not Chest Radiography in Blunt Trauma: Multicenter Prospective Cohort Study. Ann Emerg Med. 2015;66:589-600.

4. Szucs-Farkas Zsolt, Katrin Lautenschlager, Patricia M. Flach, et al. Bone images from dual-energy subtraction chest radiography in the detection of rib fractures. Eur J Radiol. 2011;79:e28-32.

5. Shelmerdine Susan C, Langan Dean, Hutchinson John C, et al. Chest radiographs versus CT for the detection of rib fractures in children (DRIFT): a diagnostic accuracy observational study. Lancet Child Adolesc Health. 2018;2:802-811.

6. Van Hise M. L., Primack S. L., Israel R. S., et al. CT in blunt chest trauma: indications and limitations. Radiographics. 1998;18:1071-1084. 
7. Pesapane Filippo, Codari Marina, Sardanelli Francesco. Artificial intelligence in medical imaging: threat or opportunity? Radiologists again at the forefront of innovation in medicine. European Radiology Experimental. 2018;2:35.

8. Sahiner Berkman, Hadjiiski Lubomir M., Chan Heang Ping, et al. Effect of CAD on radiologists' detection of lung nodules on thoracic CT scans: observer performance study. Proc. Proceedings of SPIE - The International Society for Optical Engineering, 2007, 6515:5151-5157:

9. England Joseph R., Cheng Phillip M. Artificial Intelligence for Medical Image Analysis_A Guide for Authors and Reviewers. American Journal of Roentgenology. 2019;212:1-7.

10. Shi Yinghuan, Wang Qian. The Artificial Intelligence-Enabled Medical Imaging: Today and Its Future. Chin Med Sci J. 2019;34:71-75.

11. Park Sohee, Lee Sang Min, Kim Namkug, et al. Application of deep learning-based computer-aided detection system: detecting pneumothorax on chest radiograph after biopsy. European Radiology. 2019;29:5341-5348.

12. Ciompi Francesco, Chung Kaman, van Riel Sarah J., et al. Towards automatic pulmonary nodule management in lung cancer screening with deep learning. Scientific Reports. 2017;7:46479.

13. Liang Mingzhu, Tang Wei, Xu Dong Ming, et al. Low-Dose CT Screening for Lung Cancer: Computeraided Detection of Missed Lung Cancers. Radiology. 2016;281:279-288.

14. Setio Arnaud Arindra Adiyoso, Ciompi Francesco, Litjens Geert, et al. Pulmonary Nodule Detection in CT Images: False Positive Reduction Using Multi-View Convolutional Networks. IEEE Transactions on Medical Imaging. 2016;35:1160-1169.

15. Kelly D M, Hasegawa I., Borders R., et al. High-Resolution CT Using MDCT: Comparison of Degree of Motion Artifact between Volumetric and Axial Methods. American Journal of Roentgenology. 2004;182:757-759.

16. Jin Liang, Ge Xiaojun, Lu Fang, et al. Low-dose CT examination for rib fracture evaluation. Medicine. 2018;97:e11624.

17. Sırmalı Mehmet, Tu"ru"t Hasan, Topc, u Salih, et al. A comprehensive analysis of traumatic rib fractures : morbidity, mortality and management. European Journal of Cardio-thoracic Surgery. 2003;24:133-138.

18. Talbot Brett S., Gange Christopher P., Chaturvedi Apeksha, et al. Traumatic Rib Injury: Patterns, Imaging Pitfalls, Complications, and Treatment. Radiographics. 2017;37:628-651.

19. Kim Sung Jung, Bista Anjali Basnyat, Min Young Gi, et al. Usefulness of low dose chest CT for initial evaluation of blunt chest trauma. Medicine. 2017;96:e5888.

20. Omert L., Yeaney W W, Protetch J. Efficacy of thoracic computerized tomography in blunt chest trauma. Am Surg. 2001;67:660-664.

21. Atsushi Teramoto, Tetsuya Tsukamoto, Yuka Kiriyama, et al. Automated Classification of Lung Cancer Types from Cytological Images Using Deep Convolutional Neural Networks. BioMed Research International. 2017;2017:1-6.

22. Beyer F., Zierott L., Fallenberg E. M., et al. Comparison of sensitivity and reading time for the use of computer-aided detection (CAD) of pulmonary nodules at MDCT as concurrent or second reader. Eur 
Radiol. 2007;17:2941-2947.

23. De Carvalho?Filho Antonio Oseas, Silva Aristófanes Corrêa, de Paiva Anselmo Cardoso, et al. Computeraided diagnosis system for lung nodules based on computed tomography using shape analysis, a genetic algorithm, and SVM. Med Biol Eng Comput. 2017;55:1129-1146.

24. Kahn, E. Charles. From Images to Actions: Opportunities for Artificial Intelligence in Radiology. Radiology. 2017;285:719-720.

25. SumiakiMatsumoto, YoshiharuOhno, TakatoshiAokib, et al. Computer-aided detection of lung nodules on multidetector CT in concurrent-reader and second-reader modes: A comparative study European Journal of Radiology. 2013;82:1332-1337.

26. Cheng Chi-Tung, Ho Tsung-Ying, Lee Tao-Yi, et al. Application of a deep learning algorithm for detection and visualization of hip fractures on plain pelvic radiographs. European Radiology. 2019;29:5469-5477.

27. Glomski Shahar A., R. Wortman Jeremy, W. Uyeda Jennifer, et al. Dual energy CT for evaluation of polycystic kidneys: a multi reader study of interpretation time and diagnostic confidence. Abdominal Radiology. 2018;43:3418-3424.

28. Hansmann J, Schoenberg G. M., Brix G, et al. CT of urolithiasis: comparison of image quality and diagnostic confidence using filtered back projection and iterative reconstruction techniques. Academic Radiology. 2013;20:1162-1167.

29. Cosway Ben, Mathura Narad, Mott Alison, et al. Occult Rib Fractures: Defining the Cause. Child Abuse Review. 2015;24:6-15.

30. Sha Lian Sheng, Sha Zhan Guo. Diagnosis and Analysis of Occult Rib Fracture Based on Image Postprocessing Technique. Proc. 2014 7th International Conference on Intelligent Computation Technology and Automation (ICICTA), Changsha, 2014, :198-201:

\section{Tables}

\begin{tabular}{|c|c|c|}
\hline & Characteristics & Number of Fractures \\
\hline \multirow[t]{2}{*}{ Sex } & male $(n=123)$ & $409 \llbracket 60.2 \% \square$ \\
\hline & female $(n=91)$ & $271 \llbracket 39.8 \% \rrbracket$ \\
\hline \multirow[t]{2}{*}{ Fracture types } & CRFs & $529 \otimes 77.8 \% \rrbracket$ \\
\hline & ORFs & $151 \rrbracket 22.2 \% \square$ \\
\hline
\end{tabular}


Table 2 Diagnostic performance of interns and attendings in IR and DL-CAD AR

\begin{tabular}{|c|c|c|c|c|}
\hline \multirow[t]{2}{*}{ Parameters } & & \multicolumn{2}{|c|}{ Reading modes } & \multirow[t]{2}{*}{ P value } \\
\hline & & $\mathrm{IR}$ & DL-CAD AR & \\
\hline \multirow[t]{2}{*}{ sensitivity(\%) } & Interns & 68.82 & 91.76 & $0.000 *$ \\
\hline & Attendings & 86.47 & 94.56 & $0.045^{\star}$ \\
\hline \multirow[t]{2}{*}{ specificity(\%) } & Interns & 96.25 & 97.46 & 0.621 \\
\hline & Attendings & 97.12 & 98.47 & 0.952 \\
\hline \multirow[t]{2}{*}{$\operatorname{PPV}(\%)$} & Interns & 84.50 & 93.17 & $0.034^{*}$ \\
\hline & Attendings & 93.83 & 95.67 & 0.876 \\
\hline
\end{tabular}

* the difference was statistically significant $(P<0.05)$. IR= independent reading, $D L-C A D$ AR=DL-CAD assisted reading.

Table 3 The diagnostic confidence between interns and attending radiologists with and without DL-CAD assistance

\begin{tabular}{|c|c|c|c|c|c|c|c|}
\hline \multirow[t]{2}{*}{ Readers } & & \multicolumn{5}{|c|}{ Diagnostic confidence } & \multirow{2}{*}{$\begin{array}{l}P \\
\text { value }\end{array}$} \\
\hline & & 1 & 2 & 3 & 4 & 5 & \\
\hline \multirow[t]{2}{*}{ Interns } & IR & $36(5.29 \%)$ & $61(8.97 \%)$ & 163(23.97\%) & $240(35.29 \%)$ & $180(26.47 \%)$ & \multirow[t]{2}{*}{$0.000^{*}$} \\
\hline & $\begin{array}{l}\text { DL- } \\
\text { CAD } \\
\text { AR }\end{array}$ & $6(0.88 \%)$ & $22(3.24 \%)$ & $82(12.06 \%)$ & $207(30.44 \%)$ & $363(53.38 \%)$ & \\
\hline \multirow[t]{2}{*}{ Attendings } & $\mathrm{IR}$ & $21(3.09 \%)$ & $34(5.00 \%)$ & $98(14.41 \%)$ & $226(33.24 \%)$ & $301(44.26 \%)$ & \multirow[t]{2}{*}{$0.000^{*}$} \\
\hline & $\begin{array}{l}\text { DL- } \\
\text { CAD } \\
\text { AR }\end{array}$ & $5(0.74 \%)$ & $9(1.32 \%)$ & $34(5.00 \%)$ & $200(29.41 \%)$ & $432(63.53 \%)$ & \\
\hline
\end{tabular}

\section{Figures}




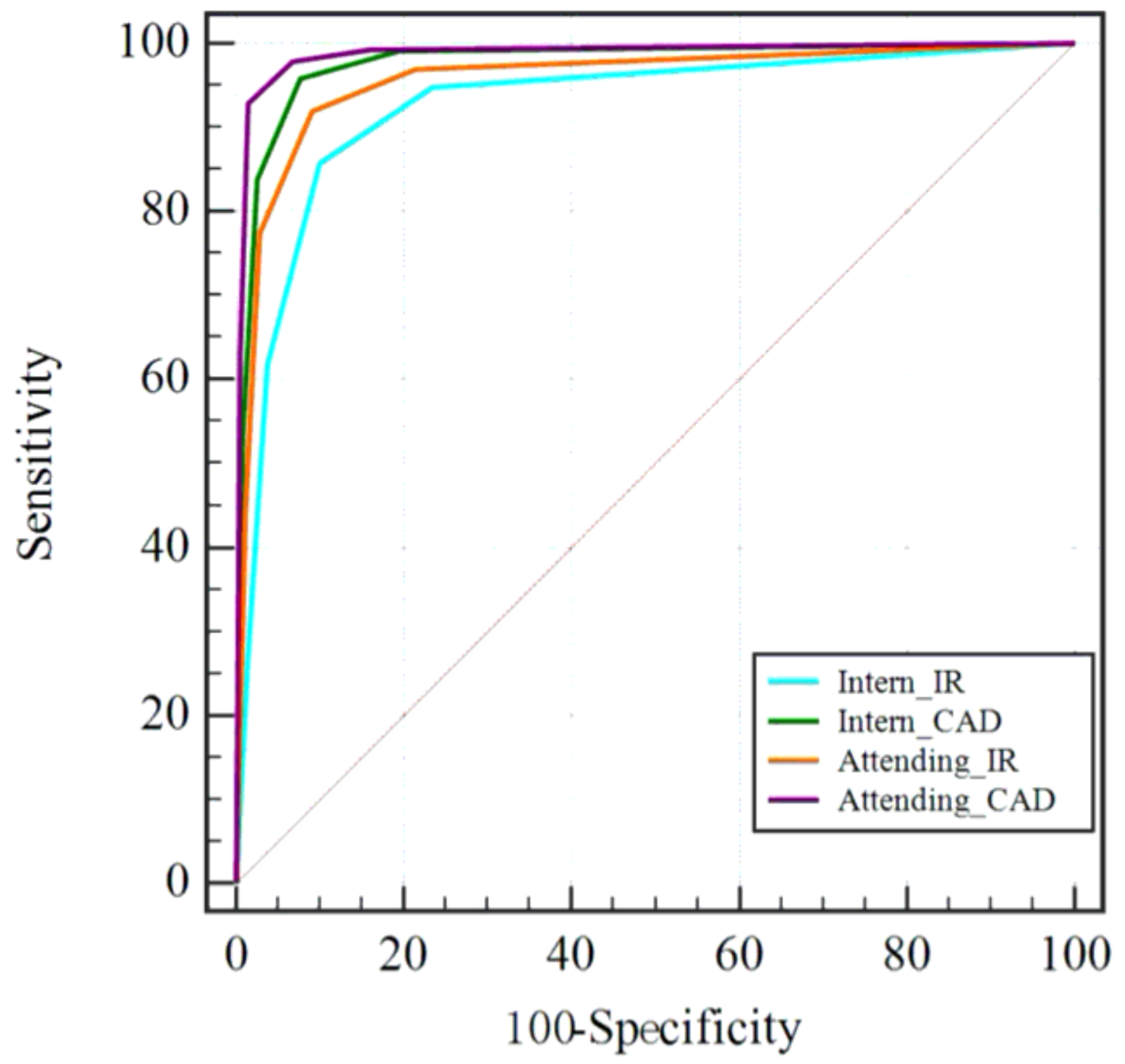

Figure 1

ROC curves for interns and attendings with and without the DL-CAD assistance. 


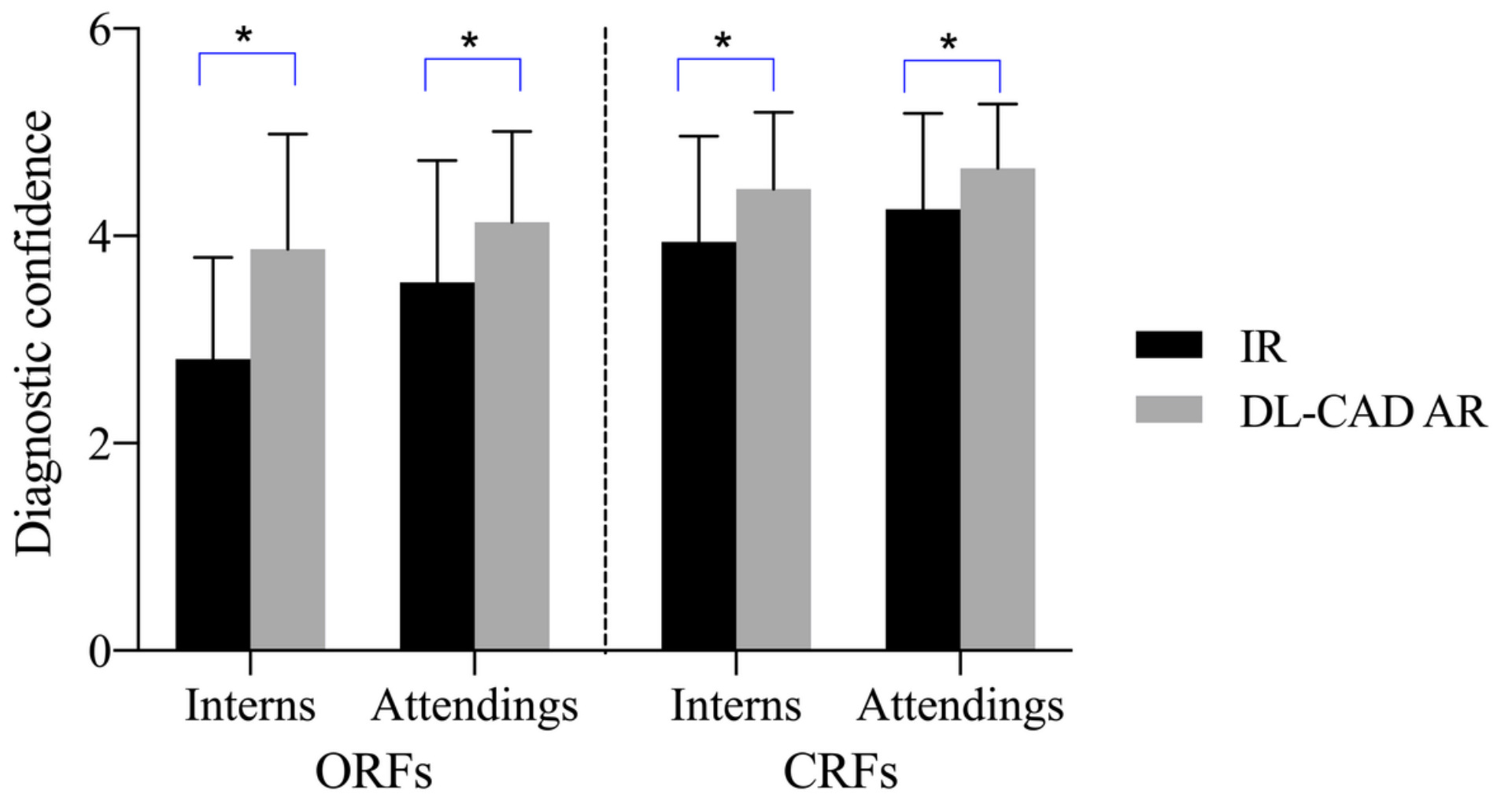

Figure 2

The comparison of diagnostic confidence of ORF and CRF between interns and attendings with and without the DL-CAD assistance. Note: * The difference was statistically significant $(P<0.05)$. IR= Independent reading, $\mathrm{DL}-\mathrm{CAD} A \mathrm{AR}=\mathrm{DL}-\mathrm{CAD}$ assisted reading, $\mathrm{ORFs=0}$ ccult rib fractures, CRFs= complete rib fractures.

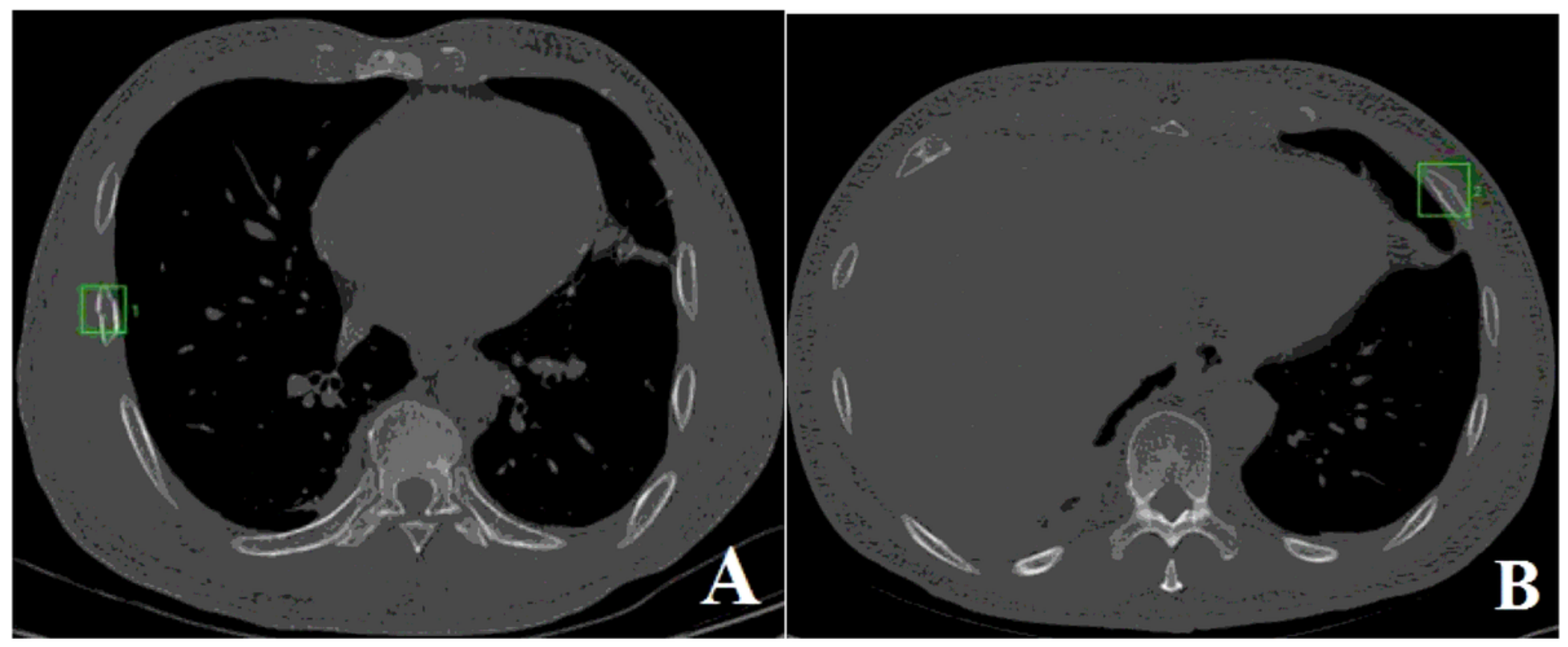

Figure 3 
A) A 49 years old male patient injured in a traffic accident. The interns and attending radiologists all accurately diagnosed rib fracture in two rounds of reading. The diagnostic confidence scores were all 5 points. With the assistance of DL-CAD, the reading time was shortened from $83 \mathrm{~s}$ and $48 \mathrm{~s}$ to $41 \mathrm{~s}$ and $28 \mathrm{~s}$, respectively. B) A 52 years old male patient with blunt chest trauma. The interns and attending radiologists missed the fracture in independent reading, and the diagnostic confidence score was 1 point. With the assistance of DL-CAD, the fracture was correctly diagnosed and the diagnostic confidence increased to 4 points.

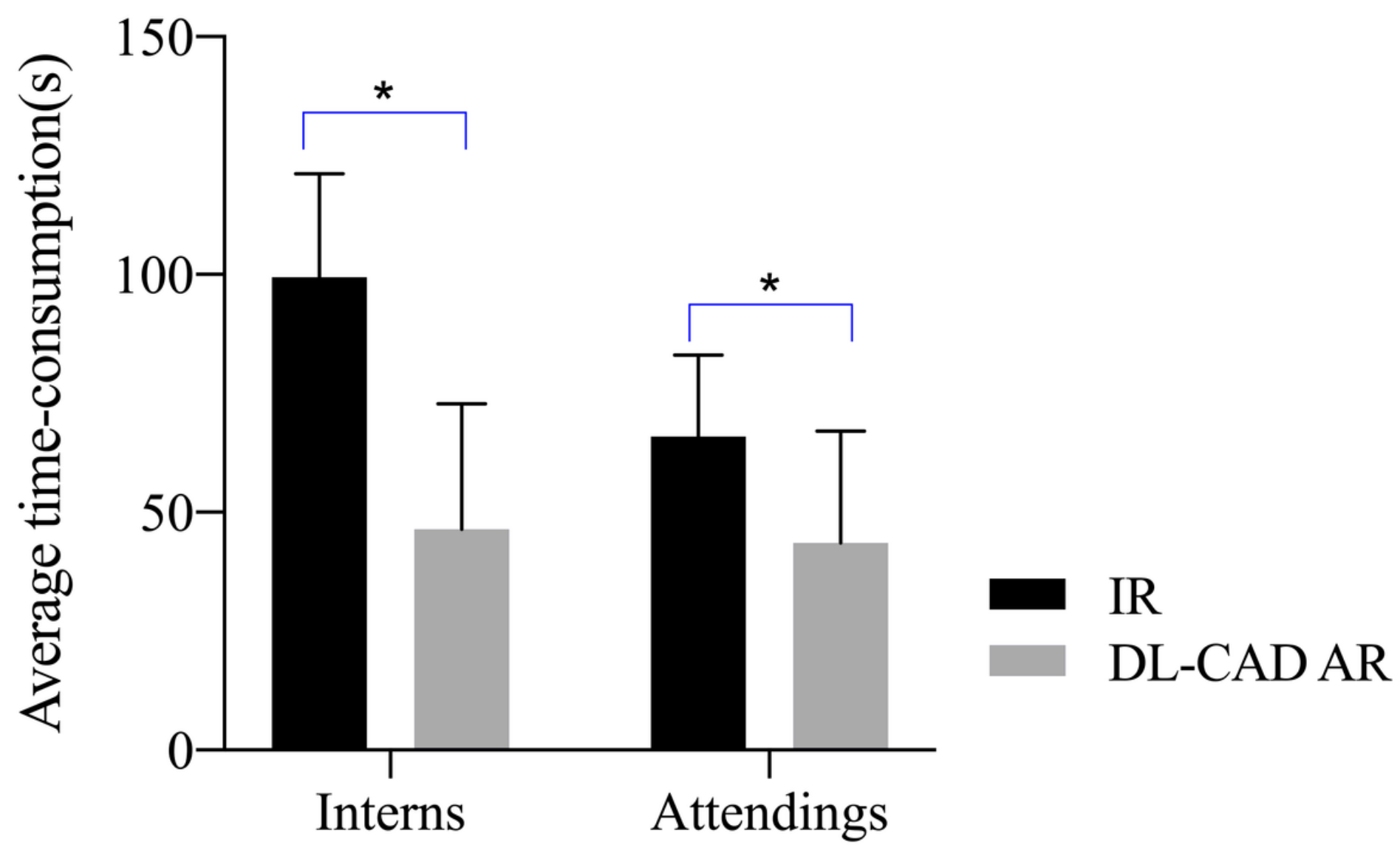

Figure 4

The average reading time of interns and attendings with and without the DL-CAD assistance. Note: * The difference was statistically significant $(P<0.05)$. 